http://dx.doi.org/10.1590/1678-4162-8711

Arq. Bras. Med. Vet. Zootec., v.68, n.6, p.1647-1654, 2016

\title{
Padrões de deslocamento de bovinos em pastos de capim-quicuiu sob lotação intermitente
}

[Displacement patterns of cattle grazing on Kikuyugrass swards under intermittent grazing]

C.H. Rocha, G.T. Santos, D.A. Padilha, D. Schmitt, C. Medeiros-Neto, A.F. Sbrissia

Universidade do Estado de Santa Catarina - Lages, SC

\begin{abstract}
RESUMO
O objetivo deste trabalho foi avaliar o padrão de deslocamento de bovinos em pastos de capim-quicuiu submetidos a diferentes alturas de entrada $(10,15,20$ e $25 \mathrm{~cm})$ e mesma proporção de rebaixamento (redução em $50 \%$ da altura inicial). O padrão de exploração das estações alimentares foi avaliado com base na observação direta de dois animais (1/observador) por unidade experimental, com concomitante registro de códigos em gravadores de voz dos passos, bocados e outras atividades. As avaliações foram realizadas duas vezes por dia (10 e 15h) durante uma hora, no primeiro e no último dia do período de ocupação dos piquetes. Adicionalmente, foram realizadas avaliações em pré e pós-pastejo da massa de forragem, massa de lâminas foliares e altura do perfilho e da bainha estendida. $\mathrm{O}$ experimento foi conduzido de acordo com um delineamento em blocos completos ao acaso, com quatro tratamento e três repetições. Durante a fase inicial de rebaixamento, as variáveis passos por minuto, número de bocados por estação alimentar e taxa de bocados foram maiores nos pastos mais baixos, reduzindo com o aumento da altura em pré-pastejo, sem diferenças significativas durante a fase final de rebaixamento. Ocorrem variações no padrão de deslocamento dos animais em pastos de capim-quicuiu durante a fase inicial de rebaixamento, quando alturas entre $20-25 \mathrm{~cm}$ apresentam melhores condições para o processo de forrageamento. O rebaixamento em $50 \%$ da altura inicial interfere negativamente no padrão de deslocamento dos animais, independentemente das metas de altura em pré-pastejo utilizadas.
\end{abstract}

Palavras-chave: Pennisetum clandestinum, severidade de desfolhação, alturas de manejo, comportamento ingestivo, estação alimentar

\begin{abstract}
The aim of this work was to evaluate the pattern of displacement of cattle grazing kikuyu grass swards at different pre-grazing heights of 10,15, 20 and $25 \mathrm{~cm}$ and submitted to grazing down to a same level of defoliation of 50\% of initial pre-grazing height. Patterns of exploitation of feed stations were evaluated from direct observation of two animals (1/observer) per experimental unit with recording of codes (steps, bites, and other activities in a portable recorder device. The evaluations were performed twice a day (10 and 15h) during one hour, at the first and the last day of stocking period. Moreover, at pre and postgrazing were evaluated: herbage mass, leaf mass and sheath, and extended tiller height. The experimental design was a complete randomized block design with three replications and four treatments. The variables steps per minute, number of bites per feeding station, and bite rate were higher in lower pastures decreasing as the sward height increased in the initial phase with no difference in the final phase of grazing down. During the initial phase of the grazing down the animals presented differences in displacement patterns, where sward heights of 20 to $25 \mathrm{~cm}$ in Kikuyu grass presented better conditions for foraging by grazing animals. Grazing down pastures to $50 \%$ of initial grazing height negatively affects displacement patterns of animals, irrespective of pre-grazing heights targets.
\end{abstract}

Keywords: Pennisetum clandestinum, Grazing down, sward height, ingestive behavior

Recebido em 1 de setembro de 2015

Aceito em 12 de maio de 2016

E-mail: sbrissia@cav.udesc.br 


\section{INTRODUÇÃO}

O capim-quicuiu (Pennisetum clandestinum Hochst ex. Chiov) é uma gramínea perene de clima tropical, originária da África, mas, devido a sua origem (regiões de altitudes acima de $1500 \mathrm{~m}$ ), é adaptada tanto a regiões tropicais quanto a subtropicais (Marais, 2001).

Essa planta é reconhecida por sua persistência, excelente resposta à adubação e irrigação, resistência ao pisoteio, bem como por ser dormente durante o período de inverno/início da primavera (Fulkerson e Lowe, 2003). Em termos produtivos, pode produzir até 30t $\mathrm{MS} / \mathrm{ha} / \mathrm{ano}$, com teores de até $17 \%$ de proteína bruta, podendo sustentar níveis de produção de até 16L/vaca sem necessidade de suplementação (Garcia et al., 2014). Nesse sentido, essa forrageira pode ser uma interessante opção para a pecuária leiteira nacional, principalmente em regiões subtropicais, onde é possível a associação com culturas de inverno (e.g. azevém anual - Lolium multiflorum L.; festuca - Festuca arundinacea Scherb.; trevo-branco - Trifolium repens L.), reduzindo, assim, os períodos de vazios forrageiros.

Conceitualmente, sistemas que objetivam maximizar a produção animal mediante a utilização da forragem pastejada devem voltar suas estratégias de manejo para a produção e o fornecimento de estruturas facilmente pastejáveis, em quantidades adequadas e sem restrições advindas do tempo de acesso dos animais às áreas destinadas ao pastejo. Em outras palavras, quando não há restrições decorrentes da oferta de forragem e/ou do tempo de acesso ao pastejo, tais estratégias devem produzir estruturas que maximizem a velocidade de ingestão de forragem (Ribeiro-Filho e Sbrissia, 2012). Nesse sentido, diversos trabalhos cujo objetivo foi compreender os fatores que determinam a velocidade de ingestão e sua relação com metas de manejo convergem para a existência de uma única altura de entrada e/ou desfolhações inferiores a $50 \%$ da altura inicial (Carvalho et al., 2001; Delagarde et al., 2000; Delagarde et al., 2001; Palhano et al., 2007; Ribeiro-Filho et al., 2009; Amaral et al., 2012; Fonseca et al., 2012; Mezzalira et al., 2014). Isso ocorre porque animais lançam mão de diferentes ajustes no comportamento ingestivo de curto prazo (massa e taxa de bocados) e no padrão de busca e exploração de estações alimentares durante o pastejo para maximizar a ingestão de forragem, até que restrições de ordem física (exploração de estrato inferiores do dossel) e quantitativa (redução de estações alimentares adequadas) ocorram, desestimulando e/ou reduzindo a ingestão instantânea de forragem (Carvalho et al., 2001; Palhano et al., 2006; Palhano et al., 2007; Ribeiro-Filho et al., 2009; Amaral et al., 2012).

Sbrissia et al. (2013) demonstraram ser possível alcançar uma mesma produtividade primária de forragem (produção, composição morfológica e valor nutritivo do material acumulado) em pastos de capim-quicuiu manejados com diferentes alturas em pré-pastejo $(15-25 \mathrm{~cm})$, desde que combinadas com desfolhações moderadas (até $50 \%$ da altura em pré-pastejo). Entretanto, não há dados que descrevam a qualidade do ambiente pastoril dentro dessas metas de manejo. O processo de procura por forragem retrata a percepção do animal quanto à qualidade e à disponibilidade do alimento no ambiente pastoril (Spalinger et al., 1988; Ungar, 1996; Palhano et al., 2006), podendo, então, ser utilizado como um critério para a criação de estruturas de forragem adequadas. Nesse sentido, a hipótese central deste trabalho é que, dentro das metas de manejo descritas por Sbrissia et al. (2013), há alterações no padrão de exploração das estações alimentares logo após a entrada dos animais nos piquetes, mas, devido a restrições de ordem estrutural do dossel (principalmente a proximidade com o solo), não haverá diferença nos padrões de deslocamento próximo ao momento de saída. Dentro desse contexto, o objetivo do presente trabalho foi determinar os padrões de deslocamento de bovinos em pastos de capim-quicuiu submetidos a diferentes alturas em pré-pastejo $(10,15,20$ e $25 \mathrm{~cm})$ combinadas a uma mesma proporção de rebaixamento $(50 \%$ da altura inicial).

\section{MATERIAL E MÉTODOS}

O trabalho foi conduzido no Setor de Bovinocultura de Leite pertencente à Universidade do Estado de Santa Catarina (CAV/UDESC), município de Lages/SC, durante os meses de abril e maio de 2013. O clima da região, de acordo com a classificação de Köppen, é $\mathrm{Cfb}$ (subtropical mesotérmico úmido), com verões amenos, ocorrência de geadas frequentes 
e temperatura média anual entre 17 e $18^{\circ} \mathrm{C}$. O pasto de capim-quicuiu foi formado no ano de 2000 pelo favorecimento da espécie que ocupava predominantemente a área e, desde então, vem sendo utilizado exclusivamente para o pastejo de bovinos leiteiros. Devido ao seu relevo (suave a moderadamente suave), a área experimental foi dividida em três blocos, cada um contendo quatro piquetes (totalizando 12 piquetes), com área média de aproximadamente $1500 \mathrm{~m}^{2}$ por piquete.

O delineamento experimental utilizado foi em blocos completos ao acaso, com três repetições. Os tratamentos corresponderam a quatro alturas em pré-pastejo $(10,15,20$ e $25 \mathrm{~cm})$, rebaixados em $50 \%$ de sua altura inicial $(5,7,5,10$ e $12,5 \mathrm{~cm}$ de altura de resíduo, respectivamente). As metas pré-pastejo foram baseadas no trabalho de Sbrissia et al. (2013), em que se verificou que, apesar de pastos de capim-quicuiu interceptarem $95 \%$ da radiação fotossinteticamente ativa em alturas próximas a $25 \mathrm{~cm}$, foi possível obter a mesma produção de forragem em alturas menores a este limite, proporcionando uma flexibilidade de utilização sem prejudicar a produção de biomassa. A proporção de desfolhação (50\% da altura de entrada) foi definida com base em trabalhos que apontam esse momento como um possível limite para a manutenção de elevadas taxas de ingestão de forragem (Delagarde et al., 2000; Delagarde et al., 2001; Ribeiro-Filho et al., 2009). As condições experimentais foram monitoradas com o uso de um bastão graduado (sward stick Barthram, 1985) em 60 pontos por unidade experimental a cada três dias, durante o período de rebrotação, e a cada 12 horas durante o período de ocupação.

Para a caracterização da estrutura do dossel forrageiro, foram efetuadas amostragens de massa de forragem e altura do perfilho estendido e bainha em pré e pós-pastejo. Para a massa de forragem, realizou-se a coleta de toda a biomassa contida no interior de amostradores quadrados com $65 \mathrm{~cm}$ de lado, em três pontos representativos da condição média (altura) da unidade experimental no momento da avaliação. Em pré-pastejo, a coleta foi estratificada em duas porções em função do tratamento da unidade experimental (denominando-as de estrato pastejável - acima do resíduo pretendido; e de resíduo pré-pastejo - do nível do solo até a altura de resíduo pretendido), com o auxílio de réguas graduadas em centímetros. No póspastejo, não houve estratificação, sendo coletado apenas o resíduo. As amostras foram, então, separadas nas frações lâminas foliares, colmo + bainhas e material senescente e, em seguida, secas em estufa a $65^{\circ}$ até o peso constante, para a obtenção do peso seco. A determinação da altura do perfilho estendido (distância entre o solo e o ápice da folha mais alta quando posicionada verticalmente) e da bainha (distância entre o solo e a última lígula exposta) se deu por meio da leitura de altura de 100 perfilhos por unidade experimental, em pré e pós-pastejo, com régua graduada em centímetros.

Os animais utilizados foram vacas secas e novilhas da raça Holandesa, com peso médio de $425 \mathrm{~kg} \pm 75 \mathrm{~kg}$, previamente treinados/ acostumados ao manejo e à presença dos avaliadores. Conforme os pastos atingiam a meta de altura inicial pretendida, os animais eram alocados aos piquetes para um prévio reconhecimento e adaptação da área onde $\mathrm{o}$ pastejo seria realizado. Durante a avaliação, dois animais por unidade experimental foram monitorados por avaliadores previamente treinados que, com gravadores de voz e utilizando códigos, registraram as seguintes variáveis: bocados (1), passos (P) e outras atividades $(\mathrm{O})$. Essas avaliações eram realizadas duas vezes por dia, uma pela manhã (às 10h) e outra no período da tarde (às $15 \mathrm{~h}$ ), com duração de uma hora para cada avaliação. As duas avaliações ocorriam no primeiro dia da fase inicial e no último dia da fase final de rebaixamento dos pastos. Assim, havia três repetições no espaço (blocos), duas repetições no tempo (avaliações de manhã e à tarde), duas repetições para cada condição (entrada e saída dos animais) e dois animais sendo avaliados simultaneamente, totalizando 96 testes de pastejo.

De posse das gravações, as seguintes variáveis foram obtidas: i) tempo total de pastejo; ii) número total de passos; iii) número total de bocados; iv) número total de estações alimentares; v) passos por unidade de tempo; vi) tempo de pastejo em cada estação alimentar; vii) tempo de permanência em cada estação alimentar; viii) passos entre estações alimentares; ix) bocados por unidade de tempo; e $\mathrm{x}$ ) número de bocados desferidos em cada estação 
alimentar. Considerou-se uma troca de estação alimentar a partir do segundo passo realizado pelo animal. O número de passos por unidade de tempo foi calculado pelo número total de passos dividido pelo tempo total de pastejo; o número de estações alimentares por unidade de tempo foi definido como o número total de estações alimentares dividido pelo tempo total de pastejo; o tempo que o animal permaneceu em cada estação alimentar foi obtido pela razão entre o tempo total de pastejo e o número total de estações alimentares; o número de passos entre estações alimentares foi estimado pela razão entre o número total de passos e o número total de estações alimentares; o número de bocados por unidade de tempo foi obtido pela divisão entre o número total de bocados e o tempo total de pastejo; e o número de bocados em cada estação alimentar pela razão entre o número total de bocados pelo número total de estações alimentares.

Os dados foram submetidos à análise de variância usando-se o PROC MIXED, do pacote estatístico SAS (Statistical Analysis System, 1999), a $10 \%$ de probabilidade, incluindo os efeitos aleatórios dos blocos e o efeito fixo das metas em pré-pastejo (alturas). A opção pelo nível de significância de $10 \%$ se deve à natureza extremamente variável desse tipo de análise
(Aurélio et al., 2007). Os efeitos linear, quadrático e cúbico das alturas foram testados utilizando-se contrastes ortogonais polinomiais.

\section{RESULTADOS E DISCUSSÃO}

Os valores de massa de forragem e de massa de folhas em pré e pós-pastejo variaram de acordo com as metas de alturas, de modo que quanto maior a altura, maiores foram os valores observados (Tab.1).

Esses resultados já eram esperados, uma vez que trabalhos concomitantes conduzidos na mesma área experimental mostraram um aumento na massa em função das alturas preestabelecidas (Sbrissia et al., 2013). Entretanto, apesar de ocorrerem aumentos na massa de folhas em póspastejo, essas diferenças foram pequenas (em média $180 \mathrm{~kg} \mathrm{MS} / \mathrm{ha})$. Isso pode ter ocorrido em função da grande proporção de coleta da massa de folhas em todos os tratamentos (em média $70 \%$ ), uma vez que animais em pastejo normalmente exercem preferência por estações alimentares com estruturas que maximizem a ingestão de nutrientes (folhas), como forma de otimizar a taxa de ingestão de nutrientes (Edouard et al., 2010; Hirata et al., 2006; Prache et al., 2006).

Tabela 1. Biomassa e massa de lâminas foliares (kg MS/ha), altura do perfilho estendido e da bainha (cm) em pré e pós-pastejo em pastos de capim-quicuiu com diferentes alturas de entrada e mesma proporção de rebaixamento (50\% da altura inicial)

\begin{tabular}{|c|c|c|c|c|c|c|c|c|}
\hline \multirow{2}{*}{ Variáveis } & \multicolumn{4}{|c|}{ Altura pré-pastejo $(\mathrm{cm})$} & \multirow{2}{*}{$\mathrm{EPM}^{5}$} & \multicolumn{3}{|c|}{ Efeito $^{4}$} \\
\hline & 10 & 15 & 20 & 25 & & Lin & Quad & $\mathrm{Cub}$ \\
\hline Biomassa pré & 2909 & 4100 & 5350 & 6025 & 573,6 & 0,007 & n.s. ${ }^{6}$ & n.s. \\
\hline Biomassa pós & 2188 & 3052 & 2943 & 3944 & 168 & 0,008 & n.s. & 0,05 \\
\hline M.L.F. ${ }^{(1)}$ pré & 1724 & 1819 & 2517 & 2532 & 194,2 & 0,013 & n.s. & n.s. \\
\hline M.L.F. ${ }^{(1)}$ pós & 334 & 678 & 577 & 695 & 52,2 & 0,072 & 0,09 & 0,04 \\
\hline A.P.E. ${ }^{(2)}$ pré & 10,8 & 21,8 & 23 & 32,5 & 2,4 & $<0,001$ & n.s. & 0,02 \\
\hline A.P.E. ${ }^{(2)}$ pós & 6,4 & 12,8 & 15,1 & 15,5 & 0,5 & 0,001 & n.s. & n.s. \\
\hline A.B. ${ }^{(3)}$ pré & 3,6 & 8,8 & 7,2 & 10 & & & & \\
\hline A.B. ${ }^{(3)}$ pós & 3,6 & 8,8 & 7,8 & 9,3 & & & & \\
\hline
\end{tabular}

${ }^{\mathrm{I}}$ Massa de lâminas foliares (kg MS/ha); ${ }^{2}$ altura do perfilho estendido (cm); ${ }^{3}$ altura da bainha $(\mathrm{cm}) ;{ }^{4}$ significativo quando $\mathrm{P}<0,10 ;{ }^{5} \mathrm{EPM}=$ erro-padrão da média; ${ }^{6}$ n.s. = não significativo.

O número de passos por minuto (P/min também interpretado como velocidade de deslocamento), na fase inicial de rebaixamento dos pastos, reduziu-se linearmente com o aumento da altura em pré-pastejo (Tab. 2). Portanto, os animais se deslocaram mais e, possivelmente, a passos mais velozes em menores alturas, o que, segundo Carvalho et al. (1999), significa uma mudança na estratégia de busca da forragem pelo animal em situações limitantes, na tentativa de aumentar a probabilidade de encontro de bocados potenciais. 
Tal mecanismo foi também citado por Palhano et al. (2006), em pastos de capim-mombaça (Panicum maximum (L) Jacq.), como uma possível resposta de pastos manejados com baixas alturas em pré-pastejo. O número de bocados por estação alimentar (B/E.A) e a taxa de bocados $(\mathrm{B} / \mathrm{min})$ reduziram-se com o aumento da altura em pré-pastejo (efeito linear; Tab. 2), comprovando novamente a interferência da estrutura do dossel sobre o processo de coleta da forragem.

Tabela 2. Padrões de deslocamento dos animais em pastejo durante a fase inicial do rebaixamento de pastos de capim-quicuiu com diferentes alturas pré-pastejo e mesma proporção de rebaixamento $(50 \%$ da altura inicial)

\begin{tabular}{|c|c|c|c|c|c|c|c|c|}
\hline \multirow{2}{*}{ Variáveis } & \multicolumn{4}{|c|}{ Altura pré-pastejo $(\mathrm{cm})$} & \multirow{2}{*}{$\mathrm{EPM}^{7}$} & \multicolumn{3}{|c|}{ Efeito $^{6}$} \\
\hline & 10 & 15 & 20 & 25 & & Lin & Quad & $\mathrm{Cub}$ \\
\hline $\mathrm{P} / \mathrm{min}^{(1)}$ & 5,2 & 5,6 & 4,5 & 4,5 & 1,3 & 0,09 & n.s. ${ }^{8}$ & n.s. \\
\hline T/E.A. ${ }^{(2)}$ & 45,9 & 39,3 & 54 & 50 & 5,7 & n.s. & n.s. & n.s. \\
\hline $\mathrm{B} / \min ^{(3)}$ & 42 & 44,6 & 36,7 & 35,2 & 2,7 & 0,02 & n.s. & n.s. \\
\hline P/E.A. ${ }^{(4)}$ & 3,9 & 3,6 & 3,5 & 3,4 & 0,3 & n.s. & n.s. & n.s. \\
\hline B/E.A. ${ }^{(5)}$ & 30,7 & 28,6 & 28,5 & 27 & 2,0 & 0,10 & n.s. & n.s. \\
\hline
\end{tabular}

${ }^{1}$ Passos por minuto; ${ }^{2}$ tempo por estação alimentar $(\mathrm{min}) ;{ }^{3}$ número de bocados por minuto; ${ }^{4}$ passos por estação alimentar; ${ }^{5}$ número de bocados por estação alimentar; ${ }^{6}$ significativo quando $\mathrm{P}<0,10$; ${ }^{7}$ erro-padrão da média; ${ }^{8}$ não significativo.

A taxa de bocados possui relação inversa com a massa do bocado, consequência da maior necessidade de movimentos mandibulares de manipulação (apreensão e mastigação) da forragem coletada com o aumento da massa do bocado (Carvalho et al., 2001; Mezzalira et al., 2014). Já o número de bocados e/ou o tempo de permanência por estação alimentar estão relacionados com a disponibilidade de nutrientes em cada estação, de modo que quanto menor a disponibilidade de nutrientes, menor é a exploração dessa estação (Palhano et al., 2006). Assim, dentro dos limites de altura em prépastejo estabelecidos para o presente experimento, o padrão de deslocamento dos animais é diferente na entrada dos piquetes, o que aparentemente aponta para uma estrutura de forragem mais acessível durante o início do período de ocupação para pastos manejados com 20 e $25 \mathrm{~cm}$

De forma geral, os padrões de deslocamento avaliados no final do período de ocupação não foram afetados pelos tratamentos impostos ( $\mathrm{P}>0,10 ;$ Tab. 3). Isso pode estar associado a questões estruturais e limitantes exercidas pelo dossel forrageiro quando estes são severamente desfolhados, refletindo em um comportamento semelhante entre os animais durante o período final de ocupação, independentemente da altura pré-pastejo.

Tabela 3. Padrões de deslocamento dos animais em pastejo durante a fase final do rebaixamento de pastos de capim-quicuiu submetidos a diferentes alturas pré-pastejo e mesma proporção de rebaixamento (50\% da altura inicial)

\begin{tabular}{|c|c|c|c|c|c|c|c|c|}
\hline \multirow{2}{*}{ Variáveis } & \multicolumn{4}{|c|}{ Altura pré-pastejo $(\mathrm{cm})$} & \multirow{2}{*}{$\mathrm{EPM}^{7}$} & \multicolumn{3}{|c|}{ Efeito $^{6}$} \\
\hline & 10 & 15 & 20 & 25 & & Lin & Quad & $\mathrm{Cub}$ \\
\hline $\mathrm{P} / \mathrm{min}^{(1)}$ & 5,3 & 4,9 & 5,0 & 5,2 & 0,4 & n.s. ${ }^{8}$ & n.s. & n.s. \\
\hline T/E.A. ${ }^{(2)}$ & 53,4 & 48,3 & 50,7 & 42,7 & 4,3 & n.s. & n.s. & n.s. \\
\hline $\mathrm{B} / \min ^{(3)}$ & 25,5 & 30,9 & 27,6 & 28,2 & 1,9 & n.s. & n.s. & n.s. \\
\hline P/E.A. ${ }^{(4)}$ & 4,6 & 3,6 & 3,9 & 3,7 & 0,3 & n.s. & n.s. & n.s. \\
\hline B/E.A. ${ }^{(5)}$ & 22,1 & 22,5 & 21,2 & 19,9 & 1,2 & n.s. & n.s. & n.s. \\
\hline
\end{tabular}

${ }^{1}$ Passos por minuto; ${ }^{2}$ tempo por estação alimentar $(\min ) ;{ }^{3}$ número de bocados por minuto; ${ }^{4}$ passos por estação alimentar; ${ }^{5}$ número de bocados por estação alimentar; ${ }^{6}$ significativo quando $\mathrm{P}<0,10 ;{ }^{7}$ erro-padrão da média; ${ }^{8}$ não significativo. 
Tal comportamento vem sendo descrito em alguns trabalhos como adaptação dos animais às estruturas impostas ao pastejo, por exemplo, a interrupção do pastejo antes do término do período de ocupação como uma forma de se condicionar à troca de piquetes, o que interfere, invariavelmente, no padrão de deslocamento (Jamieson e Hodgson, 1979; Ribeiro Filho et al., 2011; Amaral et al., 2012).

Wade (1991), ao trabalhar com azevém perene, observou uma relação direta entre a produção de leite e a altura de lâmina foliar residual. Esse autor verificou que, quando os pastos atingiam uma altura em que a altura de lâmina livre de bainha era de $7,1 \mathrm{~cm}$, a produção de leite das vacas diminuía e, então, ele associou essa redução à presença de uma barreira física para a desfolhação, ou seja, à presença de colmo e de pseudocolmo, ou à proximidade no nível do solo, dificultando, assim, o processo de apreensão e coleta da forragem. Interessante observar que, no presente trabalho, a altura de lâmina livre da bainha na fase inicial de rebaixamento para os pastos com altura de $10 \mathrm{~cm}$ já apresentava um valor de 7,2cm (Fig. 1), indicando dificuldade na apreensão de novos bocados. Já na fase final de rebaixamento, todos os tratamentos registraram valores em torno ou abaixo daquele proposto por Wade (1991), de 7,1 (Fig. 1). Além disso, Ribeiro-Filho et al., (2011) mostraram que, em pastos de azevém anual, houve redução no consumo de vacas leiteiras quando os animais pastejavam pastos com comprimento de lâmina livre de bainha de $10 \mathrm{~cm}$. Nessa perspectiva, é possível afirmar que, independentemente do tratamento testado pelo presente estudo, a fase final do rebaixamento de pastos manejados com meta de $50 \%$ de rebaixamento da altura inicial apresenta condições limitantes ao processo de captura e ingestão de forragem (o que pode explicar a semelhança no padrão de deslocamento observado entre os tratamentos e refletir em dificuldade no processo de forrageamento). Esses resultados indicam uma possível necessidade de utilizar metas de manejo menos severas quando o objetivo for beneficiar o processo de procura e coleta de forragem pelo animal.

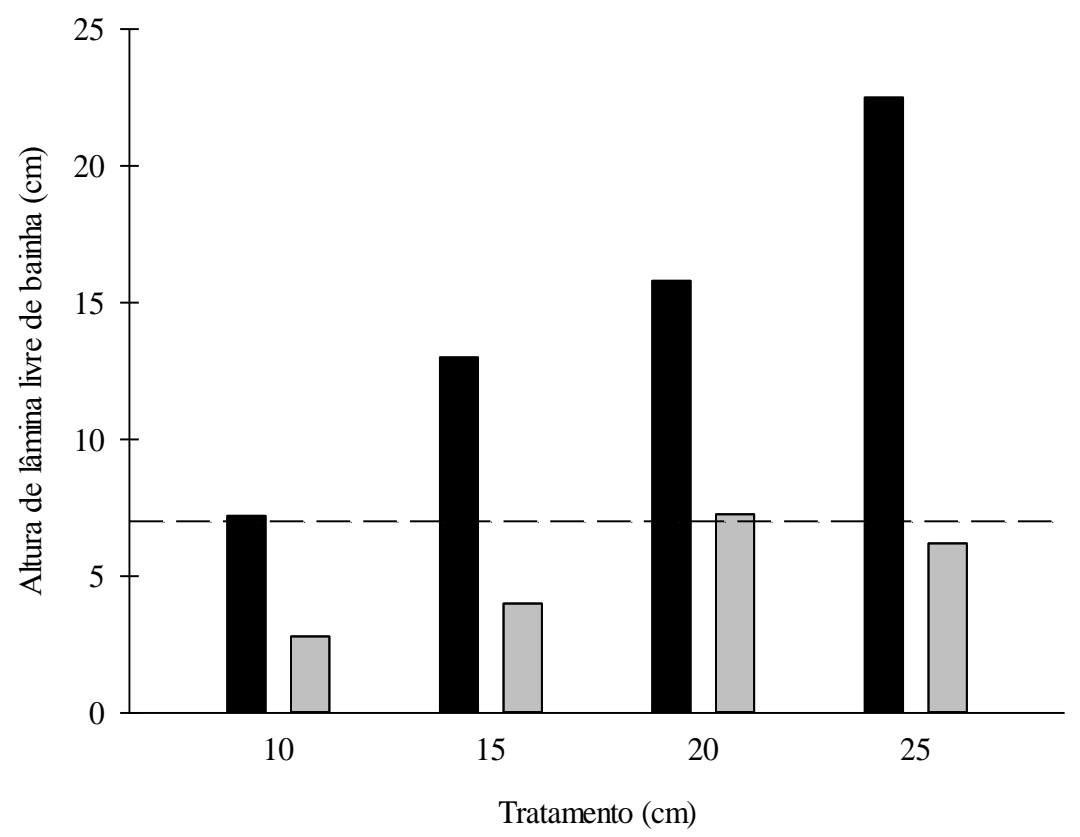

Figura 1. Altura de lâmina livre de bainha em pré (colunas em preto) e pós-pastejo (colunas em cinza) de pastos de capim-quicuiu manejado com diferentes alturas de entrada $(10,15,20$ e $25 \mathrm{~cm})$ e mesma proporção de rebaixamento (50\% da altura inicial). Linha pontilhada representa a altura de $7 \mathrm{~cm}$ proposta por Wade (1991). 


\section{CONCLUSÃO}

Ocorrem variações no padrão de deslocamento dos animais em pastos de capim-quicuiu durante a fase inicial de rebaixamento, quando alturas entre $20-25 \mathrm{~cm}$ apresentam melhores condições para o processo de forrageamento. $\mathrm{O}$ rebaixamento em $50 \%$ da altura inicial interfere negativamente no padrão de deslocamento dos animais, independentemente das metas de altura em pré-pastejo utilizadas.

\section{REFERÊNCIAS}

AMARAL, M.F.; MEZZALIRA, J.C.; BREMM, C. et al. Sward structure management for a maximum short-term intake rate in annual ryegrass. Grass Forage Sci., v.68, p.271-277, 2012.

AURÉLIO, N.D.; QUADROS, F.L.F.; MAIXNER, A.R. et al. Comportamento ingestivo de vacas holandesas em lactação em pastagens de capim- elefante anão (Pennisetum purpureum cv. Mott) e Tifton 85 (Cynodon dactylon x $C$ nlemfuensis) na região noroeste do Estado do Rio Grande do Sul. Ciênc. Rural, v.37, p.470-475, 2007.

BARTHRAM, G.T. Experimental techniques: the HFRO sward stick. In: ALCOCK, M.M. (Ed.). Biennial report of the hill farming research organization. Midlothian: Hill Farming Research Organization, 1985. p.29-30.

CARVALHO, P.C.F.; PRACHE, S.; DAMASCENO, J.C. et al. O processo de pastejo: desafios da procura e apreensão da forragem pelo herbívoro. In: REUNIÃO ANUAL DA SOCIEDADE BRASILEIRA DE ZOOTECNIA, 36., 1999, Porto Alegre. Anais... Porto Alegre: SBZ, 1999. p.253-268.

CARVALHO, P.C.F.; RIBEIRO-FILHO, H.M.N.; POLI, C.H.E.C. et al. Importância da estrutura da pastagem na ingestão e seleção de dietas pelo animal em pastejo. In: REUNIÃO ANUAL DA SOCIEDADE BRASILEIRA DE ZOOTECNIA, 38., 2001, Piracicaba. Anais... Piracicaba: SBZ, 2001. p.853-871.
DELAGARDE, R.; PEYRAUD, J.L.; DELABY, L. et al. Vertical distribution of biomass, chemical composition and pepsin-cellulase digestibility in a perennial ryegrass sward: interaction with month of year, regrowth age and time of day. Anim. Feed Sci. Technol., v.84, p.49-68, 2000.

DELAGARDE, R.; PRACHE, S.; HOUR, P.D. et al. Ingestion de l'herbe par les ruminants au pasturage. Fourrages, v.166, p.189-212, 2001.

EDOUARD, N.; DUNCAN, P.; DUMONT, B. et al. Foraging in a heterogeneous environment: an experimental study of the trade-off between intake rate and diet quality. Appl. Anim. Behav. Sci., v.126, p.27-36, 2010.

FONSECA, L.; MEZZALIRA, J.C.; BREMM, C. et al. Management targets for maximising the short-term herbage intake rate of cattle grazing in Sorghum bicolor. Livest. Sci., v.145, p.205-211, 2012.

FULKERSON, W.J.; LOWE, K.F. Perennial forage and pasture crops: establishment and maintenance. In: ROGINSKI, H.; FUQUAY, J.W.; FOX, P.F. (Eds.). Encyclopedia of dairy science. Amsterdam: Academic Press, 2003. v.2, p.1124-1131.

GARCÍA, S.C.; ISLAM, M.R.; CLARK, C.E.F. et al. Kikuyu-based pasture for dairy production : a review. Crop Pasture Sci., v.65, p.787-797, 2014.

HIRATA, M.; KANEMARU, E.; TOBISA, M. Patch choice by cattle grazing tropical grass swards: a preliminary study. Appl. Anim. Behav. Sci., v.97, p.139-144, 2006.

JAMIESON, W.S.; HODGSON, J. The effects of variation in sward characteristics upon the ingestive behaviour and herbage intake of calves and lambs under a continuous stocking management. Grass Forage Sci., v.34, p.273282, 1979.

MARAIS, J.P. Factors affecting the nutritive value of kikuyu grass (Pennisetum clandestinum): a review. Trop. Grassl., v.35, p.65-84, 2001.

MEZZALIRA, J.C.; CARVALHO, P.C.F.; FONSECA, L. et al. Behavioural mechanisms of intake rate by heifers grazing swards of contrasting structures. Appl. Anim. Behav. Sci., v.153, p.1-9, 2014. 
PALHANO, A.L.; CARVALHO, P.C.F.; DITTRICH, J.R. et al. Características do processo de ingestão de forragem por novilhas holandesas em pastagens de capim-mombaça. Rev. Bras. Zootec., v.36, p.1014-1021, 2007.

PALHANO, A.L.; CARVALHO, P.C.F.; DITTRICH， J.R. Padrões de deslocamento e procura por forragem de novilhas leiteiras em pastagem de capim-mombaça. Rev. Bras. Zootec., v.35, p.2253-2259, 2006.

PRACHE, S.; DAMASCENO, J.C. Preferences of sheep grazing down conterminal monocultures of Lolium perenne-Festuca arundinácea: test of an energy intake rate maximisation hypothesis using the short-term double weighing technique. Appl. Anim. Behav. Sci., v.97, p.206-220, 2006.

RIBEIRO-FILHO, H.M.N.; HEYDT, M.S.; BAADE, E.A.S. et al. Consumo de forragem e produção de leite de vacas em pastagem de azevém-anual com duas ofertas de forragem. Rev. Bras. Zootec., v.38, p.2038-2044, 2009.

RIBEIRO-FILHO, H.M.N.; SBRISSIA, A.F. Estratégias para o manejo dos animais e do pasto em sistemas de leite. Veterinaria, (Montevideo), v.48, Suppl., p.87-90, 2012.

RIBEIRO-FILHO, H.M.N.; SETELICH, E.A.; CRESTANI, S. et al. Relationship between diurnal grazing time and herbage intake in dairy cows in rotational grazing. Ciênc. Rural, v.41, p.2010-2013, 2011.
SBRISSIA A.F.; EUCLIDES, V.P.B.; BARBOSA, R.A. et al. Grazing management flexibility in pastures subjected to rotational stocking management: herbage production and chemical composition of kikuyu-grass swards. In: INTERNATIONAL GRASSLAND CONGRESS, 22., 2013, Sydney. Preceedings... Sydney: BGS, 2013. p.1038-1040.

SPALINGER, D.E.; HANLEY, T.A.; ROBBINS, C.T. Analysis of the functional response in foraging in the Sitka black-tailed deer. Ecology, v.69, p.1166-1175, 1988.

STATISTICAL analysis system. Version 9.1. Cary: SAS, 1999.

UNGAR, E.D. Ingestive behaviour. In: HODGSON, J., ILLIUS, A.W. (Eds.). Ecology and management of grazing systems. New York: CAB International, 1996. p.185-218.

WADE, M.H. Factors affecting the a availability of vegetative Lolium perene to grazing dairy cows with special reference to sward characteristics, stocking rate and grazing method. 1991. 89f. These (Docteur) - Universite de Rennes, Saint Gilles, FR. 